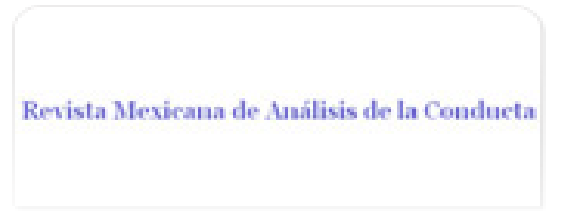

Revista Mexicana de Análisis de la Conducta ISSN: 0185-4534

editora@rmac-mx.org

Sociedad Mexicana de Análisis de la Conducta México

Torres Chávez, Álvaro Florencio; Tovar y Romo, Ángel Eugenio Efectos de nodalidad en pruebas de transferencia y de equivalencia durante la formación de clases equivalentes

Revista Mexicana de Análisis de la Conducta, vol. 34, núm. 2, diciembre, 2008, pp. 349-369 Sociedad Mexicana de Análisis de la Conducta Guadalajara, México

Disponible en: http://www.redalyc.org/articulo.oa?id=59311115012

Cómo citar el artículo

- Número completo

- Más información del artículo

Página de la revista en redalyc.org

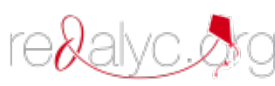

Sistema de Información Científica Red de Revistas Científicas de América Latina, el Caribe, España y Portugal Proyecto académico sin fines de lucro, desarrollado bajo la iniciativa de acceso abierto 


\title{
EFECTOS DE NODALIDAD EN PRUEBAS DE TRANSFERENCIA Y DE EQUIVALENCIA DURANTE LA FORMACIÓN DE CLASES EQUIVALENTES
}

\author{
NODALITY EFFECTS IN TRANSFERENCE AND EQUIVALENCE \\ PROBES DURING EQUIVALENCE CLASS FORMATION \\ ÁLVARO FLORENCIO TORRES CHÁVEZ \\ Y ÁNGEL EUGENIO TOVAR Y ROMO'1 \\ FACULTAD DE PSICOLOGÍA. DEPARTAMENTO DE PSICOLOGÍA \\ EXPERIMENTAL. UNIVERSIDAD NACIONAL AUTÓNOMA DE MÉXICO
}

\begin{abstract}
RESUMEN
Se analizó la presencia de efectos de "nodalidad" o del número de nodos en la formación de dos clases de estímulos equivalentes de dos nodos y cuatro miembros cada una, con un entrenamiento serial y con tres pruebas concurrentes de la formación de clases en estudiantes universitarios. Primero se entrenaron las relaciones condicionales de línea base $A B, B C$ y $C D$, luego se estableció una función discriminativa para un estímulo de cada clase potencial y se probó la transferencia de esa función hacia los otros estímulos de la clase correspondiente, después se aplicaron las pruebas tradicionales de equivalencia y por último se aplicó una prueba de relaciones derivadas en formato de "papel y lápiz". Los resultados mostraron una alta variabilidad interindividual, 4 participantes formaron las dos clases, 5 no las formaron y 1 solo formó una clase; se observaron efectos de nodalidad en 7 participantes, aunque en 5 sólo fueron parciales. Los resultados se discuten en términos de las explicaciones del efecto de nodalidad propuestas por Sidman (1994)
\end{abstract}

1. Dirigir correspondencia a: Dr. Álvaro Florencio Torres Chávez. Departamento de Psicología Experimental y Metodología. Facultad de Psicología, Universidad Nacional Autónoma de México. Av. Universidad 3004, Col. Copilco-Universidad. Cd. Universitaria, México, Distrito Federal, 04510, México. E-mail: alvarot@unam.mx.

Recibido: 23 de Noviembre, 2008. Revisado: 19 de Diciembre, 2008. Aceptado: 30 de Diciembre, 2008. 
e Imam (2006) por una parte y la de Fields y colaboradores $(1995,2008)$ por otra parte.

Palabras clave: Clases equivalentes, efectos de nodalidad, discriminación condicional, prueba de transferencia, reporte postexperimental, estudiantes universitarios.

\begin{abstract}
The presence of the "number of nodes effect" was analyzed on the formation of two-node four-member classes with a serial training protocol and with three concurrent probes of the equivalence class formation in undergraduate students. First, the baseline conditional relations $A B, B C$ and $C D$ were trained, then a discriminative function was established for one stimulus in each potential class and the transfer test was applied, finally the traditional equivalence probes and a "paper-and-pencil" probe of derivative relations were completed. The results showed a high inter-individual variability, 4 participants formed two classes, 5 not formed any and 1 alone formed one class; number of node effects was observed in 7 participants, although in 5 participants showed a partial effect. The results are discussed in terms of the Sidman's (1994) and Imam's (2006) proposals by a hand and Fields \& colleague's point of view $(1995,2008)$ on the other hand.
\end{abstract}

Key words: Equivalent classes, number of nodes effects, conditional discrimination, transfer test, post experimental report, college students.

Una clase de estímulos equivalentes es un conjunto de estímulos donde todos adquieren una función conductual común y se relacionan entre si por las propiedades de reflexividad, simetría y transitividad (Sidman \& Tailby, 1982). La importancia de la investigación del fenómeno de Equivalencia de Estímulos, desde la perspectiva del Análisis Experimental de la Conducta, radica en que constituye un modelo experimental del lenguaje, la conducta simbólica y el significado de las palabras (Dickins \& Dickins, 2001; Hayes, 1986; Mcllvane, 2000; Sidman, 1986; Sidman, 1992; Sidman, 1994). Asimismo, tiene relevancia en virtud de sus aplicaciones para la enseñanza de habilidades básicas de lectura en personas con problemas de aprendizaje y con retardo el desarrollo (Lynch \& Cuvo, 1995; Stromer, Mackay, McVay \& Fowler, 1998).

Las relaciones entre los estímulos pueden emerger sin entrenamiento explícito y directo, a partir del entrenamiento basal de discriminaciones condicionales de solo algunos miembros de la clase potencial.

Supongamos que entrenamos la relación condicional ArB y por otra parte $\mathrm{BrC}$, diremos que emerge una clase equivalente si, en una fase de prueba, 
un participante puede responder correctamente a las relaciones condicionales $\mathrm{BrA}$ y $\mathrm{CrB}$, llamadas relaciones de simetría; así como a la relación condicional ArC, llamada relación de transitividad y/o a la relación condicional CrA denominada relación de equivalencia (Sidman, 1992).

Hay varias hipótesis para dar cuenta de cómo surgen las relaciones condicionales entre estímulos que no fueron enseñadas explícitamente. Sidman (1994, 2000) menciona que las relaciones surgen como resultado del reforzamiento; por otra parte, Lowe y sus colaboradores (Dugdale \& Lowe, 1990; Horne \& Lowe, 1996) proponen que las relaciones entre estímulos se establecen por mediación del lenguaje, y que las clases equivalentes se forman cuando las personas aplican nombres comunes a los estímulos.

Lanny Fields y colaboradores (Fields, Adams, Verhave \& Newman, 1990; Fields \& Verhave, 1987; Fields, Verhave \& Fath, 1984) desarrollaron una explicación analítico-conductual alternativa de la formación de clases equivalentes. Su propuesta es importante en términos teóricos porque tiene semejanzas estructurales y funcionales con los modelos de redes de memoria semántica (Collins \& Quillian, 1969), donde los conceptos funcionan como nodos que reflejan sus interrelaciones lógicas. Fields y colaboradores señalan que las relaciones no entrenadas explícitamente entre los estímulos de una clase, resultan de una transferencia funcional determinada por uno o varios estímulos nodales y que la estructura del entrenamiento de la clase influye sobre la formación de la misma.

Un estímulo nodal es aquel que, durante la fase de entrenamiento, se relacionó con al menos otros dos estímulos (Fields, et al., 1984); por ejemplo, en las relaciones condicionales $\mathrm{ArB}$ y $\mathrm{BrC}$, el estímulo $\mathrm{B}$ es nodal debido a que está relacionado directamente con $\mathrm{A}$ y con $\mathrm{C}$. La aparición de la relación condicional $\mathrm{ArC}$, se debe al hecho de que tanto el estímulo A como el C estuvieron relacionados previamente con $\mathrm{B}$.

Como resultado del proceso de transferencia, se podría esperar que las relaciones emergentes entre estímulos en una clase sean sensibles al número de nodos con que fueron entrenadas (Fields \& Verhave, 1987; Fields, et al., 1984)

En varios estudios (Fields, Adams, Verhave \& Newman, 1990, 1993; Fields, Landon-Jimenez, Buffington \& Adams, 1995; Kennedy, 1991; Kennedy, Itkonen \& Lindquist, 1994) se ha observado un "efecto de nodalidad", consistente en que mientras más grande es el número de nodos entre estímulos relacionados, mayor es el tiempo de respuesta a esas relaciones (Bentall, Dickins \& Fox, 1993; Fields, et al., 1995; Wulfert \& Hayes, 1988).

Otra forma de medir el "efecto de nodalidad", ha considerado el momento de aparición de las relaciones emergentes; aquellas relaciones donde intervienen menos nodos aparecen primero en los bloques de prueba y las rela- 
ciones con mayor número de nodos aparecen después (Fields, et al., 1990; Kennedy, 1991; Kennedy, et al., 1994).

Se ha debatido si los efectos de nodalidad son fundamentales para entender la estructura de las clases equivalentes, el control de estímulos en una clase y el procesamiento que se tiene de los estímulos; o si más bien, estos efectos se presentan como resultado de procedimientos metodológicos inadecuados que generan malas interpretaciones (Sidman, 1994; 2000; Fields, et al., 1995; Imam, 2006).

Fields y colaboradores (1995), analizaron si los efectos producidos por el número de nodos eran transitorios o permanentes y si el efecto de nodalidad se debía a variables no controladas. Entrenaron dos clases equivalentes de 5 estímulos cada una (A, B, C, D y E), el entrenamiento fue "simultáneo", es decir, en un mismo bloque presentaron todas las relaciones de línea base ( $\mathrm{ArB}, \mathrm{BrC}, \mathrm{CrD}, \mathrm{y} \mathrm{DrE})$, para evitar efectos de nodalidad atribuibles a un entrenamiento serial; también, controlaron la cantidad de reforzamiento dado en presencia de cada estímulo discriminativo.

Los resultados indican que los 12 participantes tuvieron dificultad durante la fase de prueba para responder a las relaciones donde intervenía una mayor cantidad de nodos. Los dos participantes que formaron clases equivalentes tuvieron un responder afectado por la nodalidad, tanto en el tiempo de respuesta como en el tipo de respuesta discriminativa (número de presiones $3,5,7$ o 9 de la tecla "j") que daban en los ensayos de transferencia. Fields y colaboradores (1995) concluyeron que el efecto de nodalidad es permanente y que no se debe a variables no controladas; particularmente, no es producido ni por cantidades diferenciales de reforzamiento ni por el entrenamiento serial.

Sin embargo, los hallazgos de Fields y colaboradores (1995) fueron cuestionados por Imam (2006), quien comparó tres procedimientos de entrenamiento basal: "entrenamiento simultáneo", "entrenamiento simple a complejo" y "entrenamiento complejo a simple"; también controló la cantidad de reforzamiento dado en presencia de cada estímulo discriminativo y además uso tres estímulos de comparación. Imam no encontró que las relaciones emergentes en la fase de prueba se vieran afectadas por el número de nodos. Sus resultados apoyan la postura de que los miembros de las clases equivalentes tienen el mismo grado de relación entre si y de pertenencia a la clase; esto concuerda con la hipótesis de Sidman (1994) quién sostiene que la formación de clases equivalentes se origina en las contingencias de reforzamiento.

Lo que hace la diferencia en los tiempos de respuesta y en la exactitud de las respuestas de igualación no es el "efecto de nodalidad" o del número de nodos, sino la historia de reforzamiento (Imam, 2006), pero en particular es la historia de adquisición especifica de las relaciones condicionales de línea base la que quizá produce la diferencia. 
La mayoría de los estudios sobre "nodalidad" aplican las pruebas de equivalencia o de transferencia considerando que los participantes tienen historias de reforzamiento iguales, pero no consideran las diferencias específicas que se generan en el proceso mismo de adquisición; por lo cual el objetivo del presente estudio fue analizar el papel del proceso específico de adquisición de relaciones condicionales de línea base sobre la formación de clases equivalentes y la presencia de "efectos de nodalidad", usando el protocolo de "entrenamiento serial" pero controlando que los estímulos de prueba tuvieran valencia cero (Fields, Verhave \& Fath, 1984) y empleando tres tipos de prueba de las relaciones derivadas indicativas de la formación de clases equivalentes.

\section{MÉTODO}

Participantes

Participaron de forma voluntaria 10 estudiantes de cursos introductorios de psicología de la Universidad Nacional Autónoma de México, de género femenino, con una edad de entre 19 y 21 años (promedio 19.5 años), sin experiencia previa en tareas de equivalencia de estímulos y recibieron créditos académicos parciales por su participación.

Aparatos y Materiales

Se utilizaron computadoras marca Dell ${ }^{\circledR}$, con monitores cromáticos de 19 pulgadas, teclados y ratón de la misma marca, audífonos de diadema y formatos en papel para el reporte final. La presentación de estímulos y registro automático de respuestas se realizó mediante un programa construido en Visual Basic $6.0^{\circledR}$. La tarea experimental se realizó de forma individual en un cubículo de $3 \times 4$ metros o en un laboratorio de computación sin posibilidad de comunicación o de interferencia. La distancia entre los participantes y el monitor fue de $60 \mathrm{~cm}$.

Los estímulos fueron ocho sílabas sin sentido agrupadas de forma arbitraria en dos clases como se muestra en la Tabla 1; Las sílabas fueron de tres letras, cada letra del tipo Times New Roman ${ }^{\circledR}$ tuvo un tamaño de $1 \mathrm{~cm}^{2}$, y se mostraron en color negro sobre fondo blanco. En investigaciones sobre equivalencia de estímulos y distancia nodal con adultos, es común el uso de sílabas sin sentido para evitar sesgos de agrupamiento por el conocimiento previo del significado de las palabras; la pertenencia de las sílabas a cada clase se determinó de manera semi-aleatoria. 


\begin{tabular}{ccc}
\hline & CLASE 1 & CLASE 2 \\
\hline A & BLU & POJ \\
B & LIT & TAC \\
C & FEN & RUP \\
D & DOM & JIV \\
\hline
\end{tabular}

Tabla 1. Estímulos empleados para la formación de dos clases de estímulos equivalentes.

\section{Procedimiento}

Se realizaron dos sesiones, cada una de entre 30 y 60 minutos de duración, las sesiones fueron en días contiguos. Un experimentador dio las instrucciones de forma individual al inicio de cada fase del experimento y estuvo presente durante toda la sesión. En los ensayos de igualación de la muestra (fases 1,2 , de reentrenamiento y 5), se presentó un estímulo en la parte central superior de la pantalla, ante ese estímulo el participante oprimió la barra espaciadora, lo que produjo la aparición de otros dos estímulos de comparación en la parte inferior de la pantalla. Los participantes seleccionaron uno de los estímulos de comparación presionando las teclas "flecha izquierda" o "flecha derecha" según correspondió.

Cuando la respuesta fue correcta apareció el dibujo de una "cara feliz" durante 2 segundos en la pantalla y sonó un estímulo consistente de unas notas agudas en progresión ascendente; cuando la respuesta fue incorrecta la pantalla se oscureció en un tono gris y apareció un "tache" al centro de la pantalla por 2 segundos y sonó un estímulo consistente de dos notas graves en progresión descendente. Después de la presentación de las consecuencias hubo un intervalo entre ensayos de 1 segundo y nuevamente se presentó el estímulo de muestra. En los ensayos sin consecuencias programadas, luego de la emisión de la respuesta de selección, comenzó automáticamente el intervalo entre ensayos.

El experimento constó de cinco fases y una prueba-reporte finales que los participantes realizaron en dos sesiones, como se puede observar en la Tabla 2. 
NODALIDAD EN PRUEBAS DE TRANSFERENCIA Y DE EQUIVALENCIA

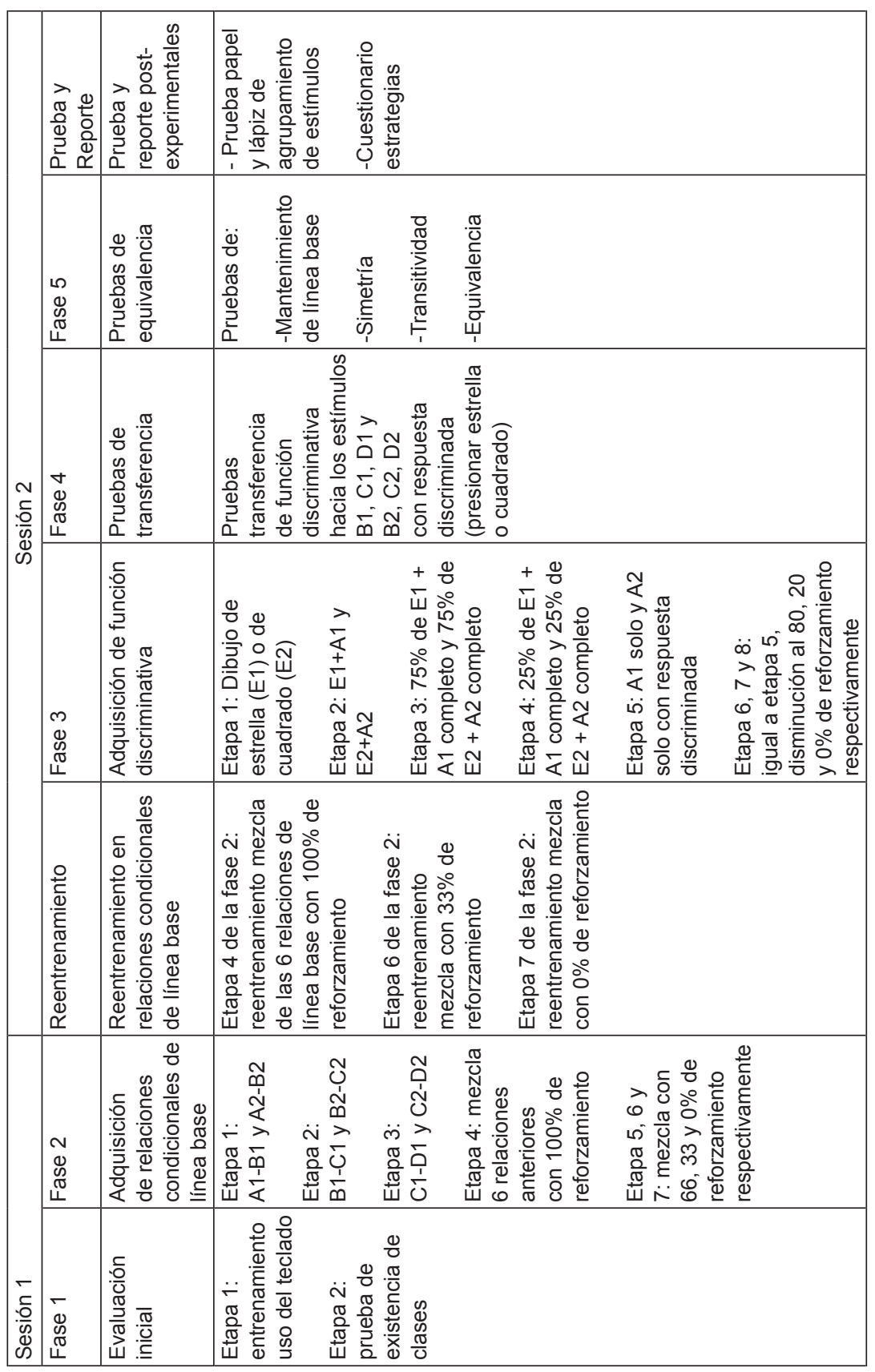

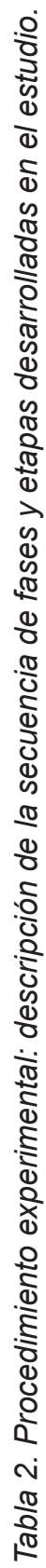


En la primera sesión se aplicaron las fases 1 y 2, que se describen enseguida:

Fase 1. Establecimiento de la forma de responder, familiarización de los participantes con el procedimiento de igualación de la muestra y evaluación de la existencia de clases. En la etapa 1 de esta fase, se presentó un bloque de 15 ensayos para enseñar la forma de responder con el teclado mediante ensayos de igualación de identidad, con estímulos ajenos a las dos clases potenciales. El criterio para esta etapa fue de un mínimo de 9 de 10 ensayos correctos en los últimos 10 ensayos del bloque. En la etapa 2 de esta fase, se presentó un bloque de 10 ensayos para evaluar el conocimiento previo de las relaciones correctas de las dos clases potenciales, no hubo criterio a cumplir.

Fase 2. En esta fase de adquisición de relaciones condicionales de línea base, se varió la posición del estímulo positivo para evitar sesgos; se varió el estímulo negativo de entre dos posibles, para evitar la selección por "exclusión"; los bloques de ensayos se diseñaron para que los estímulos tuvieran una valencia cero y para tener ensayos "válidos" en las pruebas de equivalencia (véase Fields, et al., 1984). Esta fase tuvo siete etapas de entrenamiento y de disminución del porcentaje de reforzamiento de las respuestas correctas e incorrectas.

Etapa 1: Entrenamiento de relaciones condicionales A1-B1 y A2-B2. Se presentaron bloques de 20 ensayos, el criterio fue de al menos 18 de 20 ensayos correctos; si no se logró el criterio se presentó un segundo bloque de 20 ensayos con una secuencia distinta, esto se repitió hasta el logro del criterio. Todas las respuestas tuvieron sus consecuencias correspondientes.

Etapa 2: Entrenamiento de relaciones B1-C1 y B2-C2, igual que en la etapa 1.

Etapa 3: Entrenamiento de relaciones C1-D1 y C2-D2, igual que en la etapa 1.

Etapa 4: Se presentaron bloques de 18 ensayos con una mezcla de las 6 relaciones condicionales $(A B+B C+C D)$ ya entrenadas; cada relación apareció 3 veces en cada bloque. El criterio para avanzar a la siguiente etapa fue de al menos 17 de 18 ensayos correctos.

Etapas 5, 6 y 7: disminución del reforzamiento. Se presentaron bloques de 18 ensayos con las mismas características que en la etapa 4, pero se varió la secuencia de los ensayos y se disminuyó el nivel de reforzamiento en las etapas 5,6 y 7 al $66 \%, 33 \%$ y $0 \%$ respectivamente.

En la segunda sesión se dio un reentrenamiento de la fase 2 y se aplicaron las fases 3,4 y 5 . Por último se aplicó el reporte postexperimental y la prueba de papel y lápiz (véase la Tabla 2). 
En el reentrenamiento, se presentaron nuevamente las etapas 4, 6 y 7 de la fase 2; toda vez que los participantes lograron el criterio continuaron con la siguiente fase.

En la fase 3, se estableció una función discriminativa simple para los estímulos A1 y A2 mediante un procedimiento de desvanecimiento semejante al empleado por Fields y colaboradores (1995). En la discriminación simple, los participantes oprimieron la tecla "flecha arriba", que tuvo encima el dibujo de una estrella, ante la presencia del estímulo A1, y oprimieron la tecla "flecha abajo", que tuvo encima el dibujo de un cuadrado, ante la presencia del estímulo A2.

La fase 3 tuvo ocho etapas, hubo bloques de 10 ensayos y el criterio para pasar de una etapa a otra fue de al menos 9 de 10 ensayos correctos. Las consecuencias positivas y negativas fueron las mismas que las usadas en todo el experimento y hubo un intervalo entre ensayos de 1 segundo. En las etapas 1 a 5 las respuestas generaron sus consecuencias, en las etapas 6 a 8 se disminuyó el reforzamiento. A continuación se describen las etapas de esta fase.

Etapa 1: Se presentó en el centro de la pantalla una estrella (a partir de ahora llamada E1) o un cuadrado (a partir de ahora llamado E2). Los participantes respondieron oprimiendo la tecla que tenía encima una estrella o un cuadrado.

Etapa 2: Se presentaron alineadas verticalmente en la pantalla el dibujo E1 y la sílaba A1 o bien el dibujo E2 y la sílaba A2. Cuando se presentaron E1 y A1 se reforzó la respuesta de oprimir la estrella; cuando se presentaron E2 y A2 se reforzó la respuesta de oprimir el cuadrado.

Etapa 3: Se presentaron ensayos como en la etapa anterior pero se comenzó el desvanecimiento de los estímulos $\mathrm{E}$. Se mostró sólo el $75 \%$ de los dibujos E1 o E2 y la sílaba correspondiente completa.

Etapa 4: Se presentaron ensayos como en la etapa anterior con la diferencia de que los estímulos $\mathrm{E}$ se desvanecieron aún más, se mostró sólo el $25 \%$ de los dibujos E1 o E2 y la sílaba correspondiente completa.

Etapa 5: Se presentaron ensayos como en la etapa anterior con la diferencia de que en esta etapa se eliminaron por completo los estímulos $\mathrm{E}$, por lo cual la respuesta de oprimir la tecla con la estrella o el cuadrado debía ser emitida únicamente ante $\mathrm{A} 1$ y $\mathrm{A} 2$ respectivamente.

Etapas 6, 7 y 8: Se presentaron ensayos como los descritos para la etapa 5 , con la diferencia de que se disminuyó el reforzamiento al $80 \%$ en la etapa 6 , al $20 \%$ en la etapa 7 y al $0 \%$ en la etapa 8 .

La disminución del reforzamiento tanto en la fase 2 como en la fase 3 se dispuso para establecer un nivel de ejecución elevado sin administración de consecuencias y preparar a los participantes para las fases de prueba donde ninguna respuesta es reforzada. 
Fase 4. Pruebas de transferencia de la función discriminativa (véase la Tabla 2). En esta fase se evaluó la formación de clases equivalentes a través de la transferencia de la función discriminativa establecida para los estímulos A1 y A2 hacia los miembros restantes de cada clase potencial y se midió el "efecto de nodalidad".

En esta fase hubo bloques de 32 ensayos en los que se presentó cada uno los ocho estímulos de las clases equivalentes potenciales. Ante la presencia de cada sílaba sin sentido los participantes presionaron la tecla de la estrella o la tecla del cuadrado, ninguna respuesta generó consecuencias. EI criterio de esta fase fue de al menos 31 ensayos correctos en un bloque o un total de 6 bloques.

Fase 5. Pruebas de Equivalencia. En esta fase se evaluó la formación de clases equivalentes a través de las pruebas convencionales "tipo Sidman". Hubo bloques de 40 ensayos de igualación de la muestra de los tipos de línea base, simetría, transitividad y equivalencia. Hubo ensayos de prueba adicionales para medir si los dibujos de la estrella (E1) y del cuadrado (E2) se habían incluido en las clases equivalentes. También se midió el efecto de número de nodos con relaciones de 0,1, 2 y 3 nodos. El criterio de esta fase fue al menos 38 ensayos correctos en un bloque o un total de 4 bloques.

Reporte final. Se entregó a los participantes una hoja con los ocho estímulos de las dos clases equivalentes potenciales distribuidos aleatoriamente en dos renglones y se les dio la siguiente instrucción de forma oral: "Estos son los estímulos que estuvieron apareciendo, necesito que marques de alguna forma cómo los relacionaste o cómo los agrupaste". La respuesta de los participantes constituyó una prueba de "papel y lápiz" de la formación de clases equivalentes, y fue una medida concurrente con la pruebas de transferencia y de equivalencia.

Luego se les presentó un cuestionario con las siguientes preguntas:

- ¿Cuál estrategia usaste para responder en la Fase 4? (Cuando se iban presentando todos los estímulos y debías responder a ellos con la tecla de la estrella o del cuadrado)

- ¿Cuál estrategia usaste para responder en la Fase 5? (La última Fase que realizaste, cuando tenías que seleccionar el estímulo de abajo que se relacionara con el de arriba)

- ¿Cómo aprendiste a relacionar los estímulos?

Al término de la segunda sesión, se agradeció a los participantes su colaboración y se les indicó que posteriormente serían informados sobre los propósitos y resultados del experimento. 


\section{RESULTADOS}

Se puede observar que los datos indican una alta variabilidad entre participantes en cuanto al logro de los criterios de las pruebas de transferencia, de las pruebas de equivalencia y en cuanto a la presencia del efecto de nodalidad.

\begin{tabular}{cccc}
\hline Participante & $\begin{array}{c}\text { Criterio de } \\
\text { transferencia }\end{array}$ & $\begin{array}{c}\text { Criterio de } \\
\text { equivalencia }\end{array}$ & $\begin{array}{c}\text { Efecto de } \\
\text { nodalidad }\end{array}$ \\
\hline $\mathbf{1 0 1}$ & SI & SI & No \\
$\mathbf{1 0 7}$ & SI & SI & No \\
102 & SI & SI & parcial \\
103 & SI & SI & parcial \\
111 & NO & SI & No \\
104 & NO & NO & parcial \\
105 & NO & NO & parcial \\
106 & NO & NO & parcial \\
$\mathbf{1 1 0}$ & NO & NO & Si \\
$\mathbf{1 1 2}$ & NO & NO & Si \\
\hline
\end{tabular}

Tabla 3. Logro de criterios en las pruebas de transferencia y equivalencia, así como la presencia-ausencia del efecto del nodalidad (en negritas se señalan los participantes seleccionados para un análisis detallado de su historia de reforzamiento).

En la Tabla 3 se observa que 4 participantes lograron los criterios de las pruebas de transferencia y de equivalencia, dos de ellos no mostraron efecto de nodalidad y los otros dos mostraron un efecto parcial (sólo entre algunos estímulos, o sólo entre algunas relaciones derivadas o sólo en una clase); asimismo, el participante 111 sólo logró el criterio en las pruebas de equivalencia y no mostró efecto de nodalidad; y también se observa que otros 5 participantes no lograron el criterio ni en las pruebas de transferencia ni en las pruebas de equivalencia, 3 de ellos mostraron efecto parcial de nodalidad y los otros 2 mostraron un efecto de nodalidad.

Dada la diversidad de los resultados, seleccionamos a los 4 participantes ubicados en los extremos de presencia-ausencia de nodalidad para realizar un análisis detallado de sus datos. Los 4 participantes seleccionados finalizaron el experimento en dos sesiones; los participantes 101 y 107 si lograron los criterios de las pruebas de transferencia y de equivalencia y no mostraron efecto de nodalidad; los participantes 110 y 112 no lograron los criterios de dichas pruebas y mostraron efectos de nodalidad (véase Tabla 3). 
En la fase 1, de evaluación inicial, estos cuatro participantes tuvieron un número de respuestas acertadas que estuvo cerca del nivel de azar. Enseguida presentamos los resultados de cada uno de los participantes seleccionados.

Participante 101. Durante la fase 2 (entrenamiento de relaciones condicionales de línea base), este participante aprendió las relaciones A-B en 4 bloques, las relaciones $B-C$ en 2 bloques y las relaciones $C-D$ en 1 bloque. Logró el criterio de las relaciones mezcladas $A B+B C+C D$ con disminución del reforzamiento en 4 bloques. La disminución del nivel de reforzamiento no afectó su nivel de ejecución.

El criterio del reentrenamiento lo logró en 3 bloques de ensayos. En la fase 3 (adquisición de la función discriminativa de los estímulos A1 y A2), cumplió en 8 bloques de ensayos (véase la Tabla 4).

En la fase 4 (prueba de transferencia) respondió a los 32 ensayos del primer bloque de forma correcta, esto indica que la función discriminativa de los estímulos A1 y A2 se transfirió al resto de los estímulos de cada clase, el tiempo promedio de respuesta durante esta fase no revela efectos de nodalidad (véase la Figura 1).

En la fase 5 (pruebas de equivalencia), respondió 38 de 40 ensayos de forma correcta y en 1 bloque alcanzó el criterio para terminar la fase. En la Figura 2, se muestra el tiempo promedio de respuesta de este participante para cada tipo de relación, no se aprecian efectos de nodalidad. En la Tabla 5 se muestra el porcentaje de errores de este participante según el tipo de relación, tampoco se observan efectos de nodalidad.

Participante 107. Durante la fase 2 (entrenamiento de relaciones de línea base), este participante adquirió las relaciones condicionales $A-B$ y B-C en 2 bloques cada una, la relación C-D la adquirió en 1 bloque. Logró el criterio de las relaciones mezcladas $A B+B C+C D$ con disminución del reforzamiento en 6 bloques. La disminución del reforzamiento no afectó su nivel de ejecución (véase la Tabla 4).

Este participante logró los criterios del reentrenamiento en 5 bloques. En la fase 3 (adquisición de la función discriminativa de los estímulos A1 y A2), cumplió el criterio en 8 bloques de ensayos, tuvo una sola respuesta errónea (véase la Tabla 4). 


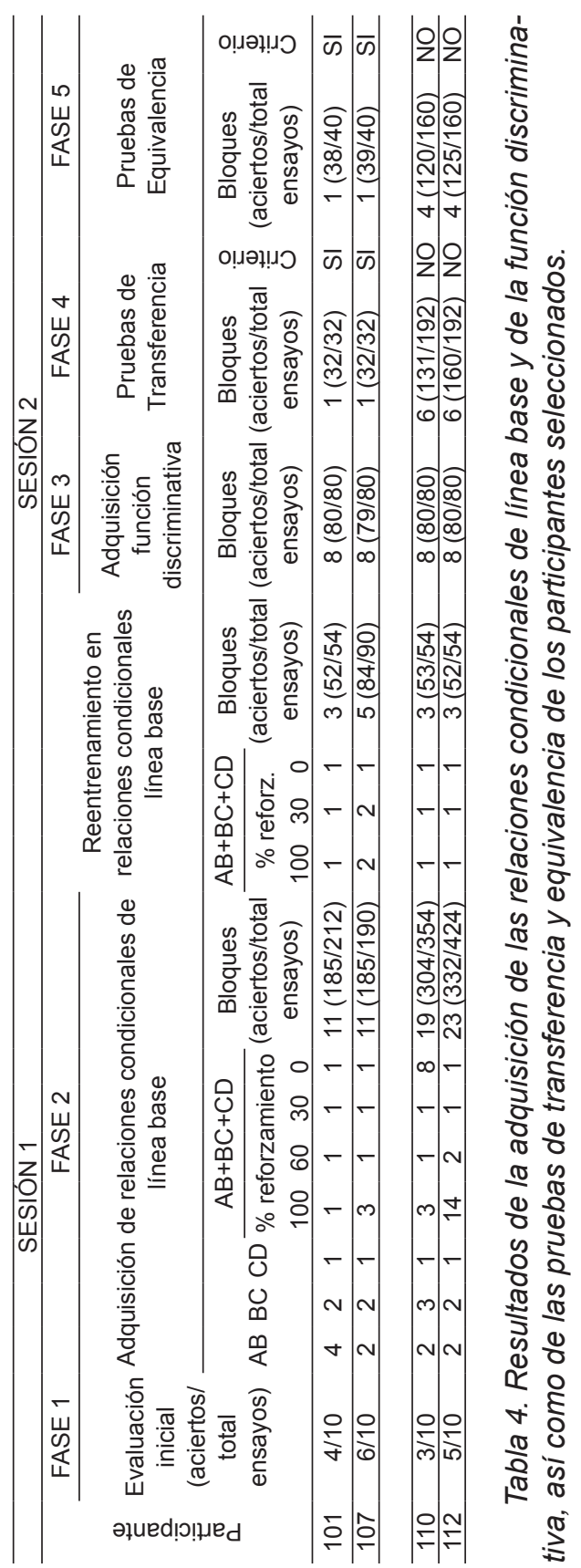


En la fase 4 (prueba de transferencia) obtuvo todos los aciertos correctos terminando en 1 bloque y mostrando una transferencia en ambas clases, al igual que el participante 101 no muestra efectos de nodalidad en el tiempo promedio de respuesta (véase la Figura 1).

En la fase 5 (pruebas de equivalencia) terminó en 1 bloque con 39 de 40 ensayos correctos, este participante tampoco muestra efectos de nodalidad en los tiempos de respuesta (véase Figura 2) ni en el porcentaje de errores dependiendo de la cantidad de nodos implicados (véase la Tabla 5).
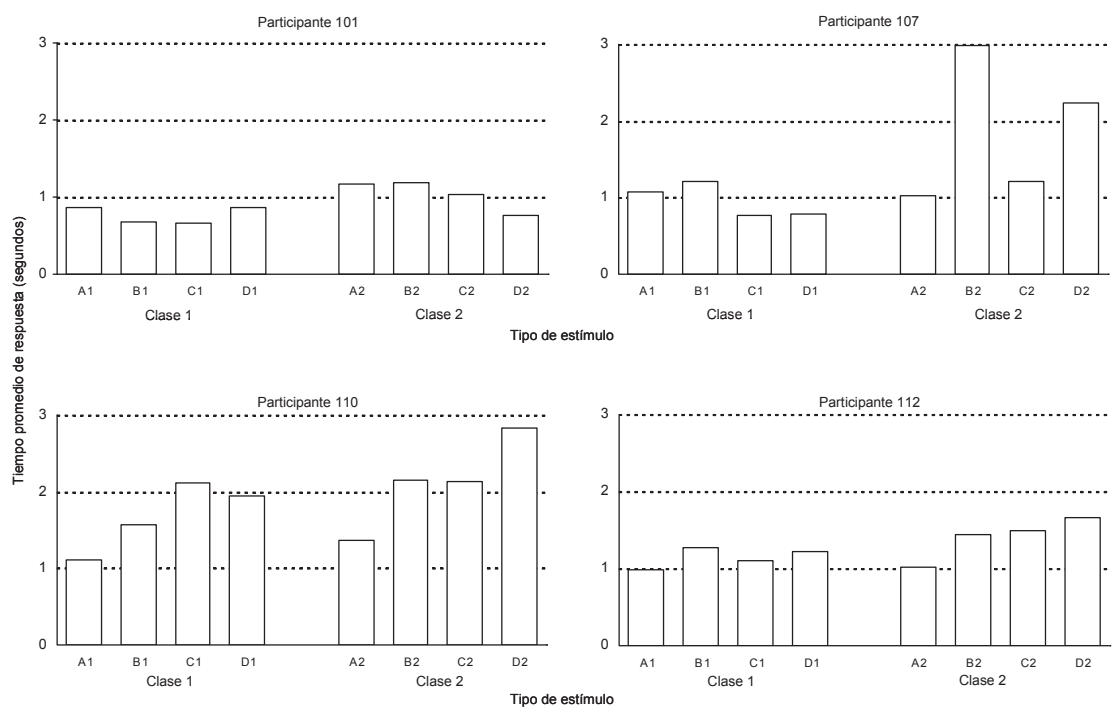

Figura 1. Tiempo de respuesta promedio en las pruebas de transferencia para cada uno de los estímulos de las dos clases potenciales. 


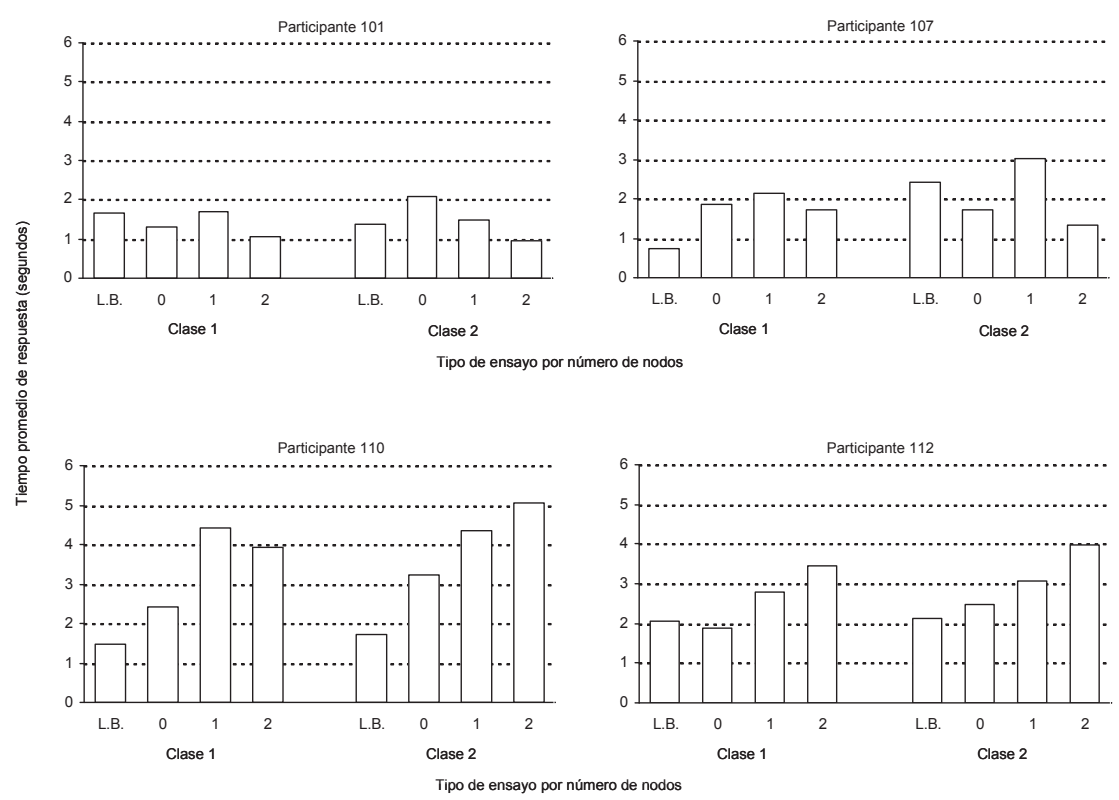

Figura 2. Tiempo promedio de respuestas en las pruebas de equivalencia de acuerdo con el número de nodos de cada prueba.

Participante 110. Durante la fase 2 (adquisición de relaciones condicionales de línea base) este participante logró el criterio de adquisición de las relaciones condicionales $\mathrm{A}-\mathrm{B}$ en 2 bloques de ensayos, luego se tardó 3 bloques de ensayos para aprender la relación $B-C$, y le tomó 1 bloque adquirir la relación $C-D$. Logró el criterio de las relaciones mezcladas $A B+B C+C D$ con disminución del reforzamiento en 13 bloques; cuando el reforzamiento se eliminó totalmente ( $0 \%$ ), se tardó 8 bloques de ensayos (véase la Tabla 4).

El participante 110 logró los criterios del reentrenamiento en el mínimo posible de 3 bloques de ensayos. En la fase 3 (adquisición de la función discriminativa de los estímulos A1 y A2), también cumplió el criterio en 8 bloques (véase la Tabla 4).

En la fase 4 (prueba de transferencia), este participante terminó 6 bloques de ensayos sin lograr el criterio, por lo cual la fase se concluyó. En la Figura 1 se puede apreciar que muestra efectos de nodalidad en el tiempo 
de respuesta; los tiempos son mayores ante estímulos con mayor número de nodos respecto de los estímulos A1 y A2, excepto para el estímulo D de la clase 1.

En la fase 5 (pruebas de equivalencia), este participante realizó 4 bloques de ensayos sin alcanzar el criterio, por lo que se finalizó la fase. En la Figura 2, se observa el efecto típico de nodalidad en su clase 2, donde el tiempo promedio aumenta conforme aumenta el número de nodos. En el porcentaje de errores por tipo de relación "nodal", también se aprecia un efecto de nodalidad; el porcentaje de error se eleva conforme aumenta el número de nodos (véase la Tabla 5).

\begin{tabular}{ccccc}
\hline & \multicolumn{4}{c}{ Porcentaje de errores } \\
\hline Tipo de ensayo & 101 & 107 & 110 & 112 \\
LB & 16.7 & 0.0 & 8.3 & 12.5 \\
N.N.0 & 0.0 & 0.0 & 15.0 & 12.5 \\
N.N.1 & 8.3 & 8.3 & 16.7 & 14.6 \\
N.N.2 & 0.0 & 0.0 & 43.8 & 40.6 \\
N.N.3 & 0.0 & 0.0 & 62.5 & 37.5 \\
\hline
\end{tabular}

$\mathrm{N} . \mathrm{N}$. = número de nodos

Tabla 5. Porcentaje de errores en las pruebas de equivalencia por tipo de ensayo.

Participante 112. Durante la fase 2 (adquisición de relaciones condicionales de línea base) este participante logró el criterio de adquisición de las relaciones condicionales A-B en 2 bloques de ensayos, luego se tardó 2 bloques de ensayos para aprender la relación $\mathrm{B}-\mathrm{C}$, y le tomó 1 bloque adquirir la relación $C-D$. Logró el criterio de las relaciones mezcladas $A B+B C+C D$ con disminución del reforzamiento en 18 bloques, cuando se le presentaron por primera vez las relaciones mezcladas tardó 14 bloques para lograr el criterio (véase la Tabla 4).

Este participante logró los criterios del reentrenamiento en el mínimo posible de tres bloques de ensayos. En la fase 3 (adquisición de la función discriminativa de los estímulos A1 y A2), también cumplió el criterio en el mínimo de 8 bloques de ensayos (véase la Tabla 4).

En la fase 4 (prueba de transferencia), este participante terminó 6 bloques de ensayos sin lograr el criterio, por lo cual la fase se concluyó. En la Figura 1 se puede apreciar que tuvo efectos de nodalidad en el tiempo de respuesta en las dos clases equivalentes potenciales; aunque el tiempo de 
respuesta al estímulo B1 es mayor que los tiempos de los demás estímulos de la clase 1.

En la fase 5 (pruebas de equivalencia), el participante 112 realizó 4 bloques de ensayos sin alcanzar el criterio, por lo que se finalizó la fase. En la Figura 2, se aprecian efectos de nodalidad en los tiempos promedio de respuesta, en su clase 1 se observa el efecto de nodalidad, aunque el tiempo de respuesta es mayor para los ensayos de línea base que para los de 0 nodos; en su clase 2 se observa el patrón típico del efecto de nodalidad. En el porcentaje de errores por tipo de relación "nodal", también se aprecia un efecto de nodalidad, el porcentaje de error se eleva conforme aumenta el número de nodos implicados (véase la Tabla 5).

Reporte postexperimental. El contenido del reporte postexperimental de los participantes 101 y 107 coincidió con los resultados de sus fases 4 y 5 , ya que indica la formación de clases equivalentes, estos participantes escribieron los estímulos agrupándolos en dos clases. Los participantes 110 y 112 no muestran agrupamiento de los estímulos consistente con las clases.

En las respuestas de los participantes a las preguntas del reporte postexperimental, destaca que los participantes 101 y 107 dan cuenta de la existencia de dos clases. El participante 110 mencionó haber "formado grupos", pero no especificó cuántos grupos ni cómo los formó. El participante 112 mencionó que fue "haciendo cadenas de estímulos", lo cual es acorde con la observación de efectos de nodalidad.

\section{DISCUSIÓN}

Los resultados del presente estudio muestran que los participantes tuvieron un patrón de ejecución experimental muy variado: hubo quienes formaron dos clases, sólo una y quienes no formaron las clases equivalentes; hubo participantes que tuvieron efecto de nodalidad, efecto parcial de nodalidad y quienes no tuvieron efecto de nodalidad. Esta variabilidad ocurrió a pesar de que se aplicó a los participantes el mismo procedimiento de entrenamiento serial de las relaciones de línea base y a pesar de que el experimento estuvo planeado para controlar la valencia de los estímulos, la valencia de cada estímulo es 0 si los participantes hubiesen logrado los criterios en el primer bloque, pero si requirieron de más bloques la valencia cambió. Esto puede explicar algunas de las diferencias individuales.

Comúnmente los efectos de nodalidad se analizan en participantes que han formado clases equivalentes y se da una menor importancia a la presencia de estos efectos en los participantes que no logran la formación de clases. En este estudio analizamos ambos tipos de participantes. 
Las ejecuciones de los participantes 101 y 107 en las pruebas de transferencia, de equivalencia y post-experimental indican que si formaron las dos clases equivalentes sin mostrar efectos de nodalidad. Estos datos apoyan la idea sustentada por Sidman (1994, 2000) e Imam (2006), de que el efecto de nodalidad contradice la idea misma de clase equivalente y de que los efectos de nodalidad se deben a un artefacto metodológico del tipo de entrenamiento o prueba de las relaciones condicionales.

Por otra parte, las ejecuciones de los participantes 110 y 112 indican que no formaron las clases equivalentes pero si tuvieron efectos de nodalidad tanto en las pruebas de transferencia como en las de equivalencia. Estos resultados apoyan la propuesta de Fields y colaboradores (Fields, Adams, Verhave \& Newman, 1990; Fields, Landon-Jimenez, Buffington \& Adams, 1995; Fields \& Moss, 2008; Fields, Verhave \& Fath, 1984) quienes afirman que, dependiendo del número de nodos existentes entre los estímulos durante el entrenamiento de línea base, estos adquieren diferentes "grados de relacionalidad" (relatedness) entre si y distintos grados de pertenencia a la clase. Asimismo, afirman que los estímulos de una clase equivalente controlan de modo diferencial la conducta asociada con la misma. Los datos generales no apoyan de modo concluyente ninguna de las dos posturas recién mencionadas.

Tal vez los resultados, distintos y variados entre los participantes seleccionados, se debieron a la interrupción de las relaciones condicionales de línea base por la introducción del entrenamiento de una discriminación simple y su correspondiente prueba de transferencia.

Sin embargo, los datos indican que una posible explicación se encuentra en la adquisición misma de las relaciones condicionales de línea base, donde se puede observar que la introducción de la mezcla de relaciones $A B+B C+C D$, en la etapa 4 de la fase 2, produjo que los participantes 107 , 110 y 112 incrementaran a 3, 3 y 14 respectivamente la cantidad de bloques que necesitaron para el logro del criterio, aun cuando en la etapa inmediata anterior lo habían conseguido en 1 bloque de ensayos.

Otro factor de esta hipótesis, sobre la importancia del proceso de adquisición de las relaciones condicionales de línea base, proviene de la eliminación del reforzamiento; tal y como se observó en el caso del participante 110, quien sólo pudo lograr el criterio de la etapa 7 de la fase $2(A B+B C+C D$ con $0 \%$ de reforzamiento) hasta que completó 8 bloques de ensayos.

En este estudio aplicamos tres pruebas para evaluar la formación de clases equivalentes: primero una prueba de transferencia de una función discriminativa simple, establecida solo para uno de los estímulos de cada clase; luego las pruebas habituales de las propiedades indicativas de equivalencia de estímulos (simetría, transitividad y equivalencia); y después una prueba post-experimental de papel y lápiz. Las tres pruebas generaron datos consistentes respecto de la formación de dos clases equivalentes. 
En las pruebas de equivalencia mezclamos ensayos de línea base con los de simetría, transitividad y equivalencia, y agregamos algunos ensayos para probar si el dibujo de la estrella y del cuadrado que usamos para establecer una respuesta discriminada habían pasado a formar parte de las clases equivalentes, los participantes 101 y 107 tuvieron correctos los ensayos donde se incluyeron estos estímulos; sin embargo, se requiere un análisis más detallado de esta inclusión de nuevos miembros a las clases en estudios futuros.

La mayoría de las investigaciones sobre equivalencia de estímulos y en particular sobre los efectos de nodalidad, analizan y discuten sus hipótesis con base en los datos de niveles de ejecución (tiempo de respuesta y/o número de errores). En esta investigación aplicamos además un reporte escrito postexperimental como medida concurrente, que permitió indagar sobre otros determinantes de la ejecución de los participantes; por ejemplo, analizamos si los participantes reportaron verbalmente la existencia de las dos clases. Asimismo, observamos cómo los participantes agruparon los estímulos cuando no se les impuso ninguna restricción (solo se les dijo que indicaran "de alguna forma" cómo habían agrupado o relacionado los estímulos); los participantes 101 y 107 agruparon por escrito los estímulos en dos clases, mientras que los participantes 110 y 112 indicaron las clases con líneas, pero lo hicieron de forma incompleta y/o no correcta.

La variabilidad de los resultados del presente estudio señalan la necesidad de profundizar la investigación sobre el papel que el número de nodos entre estímulos de entrenamiento tiene sobre la formación de clases equivalentes; hay preguntas que requieren de respuestas claras, por ejemplo: ¿el efecto de nodalidad es real o es un artefacto experimental?, negar la existencia del efecto de nodalidad o disminuir su manifestación a través de controles experimentales no responde la pregunta. En el caso de nuestro estudio, surgen preguntas acerca del ¿porqué hubo participantes que formaron clases equivalentes y no mostraron nodalidad y viceversa?, ¿son procesos conductuales independientes?

El estudio profundo de estos fenómenos repercutirá en las áreas de formación de conceptos y significados; así como en el entendimiento de distintos tipos de clases equivalentes, con o sin nodalidad, y su ubicación en escenarios naturales de aprendizaje.

Se requiere de investigaciones donde se afinen las formas de medición de efectos de nodalidad, una limitación del presente estudio es que las latencias de respuesta pueden variar debido a factores que no controlamos, tales como cansancio y/o distracción de los participantes; de ahí también la importancia de tener varias medidas concurrentes, como cantidad de errores dependiendo del tipo de relación nodal y promedios de latencia de respuesta en las prueba de transferencia y de equivalencia. 


\section{REFERENCIAS}

Bentall, R. P., Dickins, D. W., \& Fox, S. R. A. (1993). Naming and equivalence: Response latencies for emergent relations. Quarterly Journal of Experimental Psychology, 46B, 187-214.

Collins, A. M. \& Quillian, M. R. (1969). Retrieval time from semantic memory. Journal of Verbal Learning and Verbal Behavior, 8, 240-247.

Dickins, T., Dickins, D. (2001) Symbols, stimulus equivalence and the origins of language. Behavior and Philosophy, 29, 221-244.

Dugdale, N. \& Lowe, C. F. (1990). Naming and stimulus equivalence. En D. E. Blackman \& H. Lejeune. (Eds.) Behavior Analysis in Theory and Practice: Contributions and controversies. (pp. 115-138). Hove, England: Erlbaum.

Fields, L., Adams, B., Verhave, T., \& Newman, S. (1990). The effects of nodality on the formation of equivalence classes. Journal of the Experimental Analysis of Behavior, 53, 345-358.

Fields, L., Adams, B., Verhave, T., \& Newman, S. (1993). Are stimuli in equivalence classes equally related to each other? The Psychological Record, 45, 85-105.

Fields, L., Landon-Jimenez, D., Buffington, D. \& Adams, B. (1995). Maintained nodaldistance effects in equivalence classes. Journal of the Experimental Analysis of Behavior, 64, 129-145.

Fields, L. \& Moss, P. (2008). Formation of partially and fully elaborated generalized equivalence classes. Journal of the Experimental Analysis of Behavior, 90, 135168.

Fields, L., \& Verhave, T. (1987). The structure of equivalence classes. Journal of the Experimental Analysis of Behavior, 48, 317-332.

Fields, L., Verhave, T., \& Fath, S. (1984). Stimulus equivalence and transitive associations: A methodological analysis. Journal of the Experimental Analysis of Behavior, 42, 143-157.

Hayes, S. C. (1986). The case of the silent dog - Verbal reports and the analysis of rules: A review of Ericsson and Simon's protocol analysis: Verbal reports as data. Journal of the Experimental Analysis of Behavior, 45, 351-363.

Horne, P. J. \& Lowe, C. F. (1996). On the origins of naming and other symbolic behavior. Journal of the Experimental Analysis of Behavior, 65, 185-241.

Imam, A. (2006). Experimental control of nodality via equal presentations of conditional discriminations in different equivalence protocols under speed and no-speed conditions. Journal of the Experimental Analysis of Behavior, 85, 107-124.

Kennedy, C. L. (1991). Equivalence class formation influenced by the number of nodes separating stimuli. Behavioral Processes, 24, 219-245.

Kennedy, C. L., Itkonen, T., \& Lindquist, K. (1994). Nodality effects during equivalence class formation: An extension to sight-word reading and concept development. Journal of Applied Behavior Analysis, 27, 673-683.

Lynch, D. \& Cuvo, A. (1995). Stimulus equivalence instruction of fraction-decimal relations. Journal of Applied Behavior Analysis, 28, 115-126. 
Mcllvane, W. (2000). Methods for studying symbolic behavior and category formation: contributions of stimulus equivalence research. Developmental Review, 21, 355374.

Sidman, M. (1986). Functional analysis of emergent verbal classes. En T. Thompson \& Zeiler, M. D. (Eds.). Analysis and integration of behavioral units. (pp. 213-245). Hillsdale, NJ: Lawrence.

Sidman, M. (1992) Equivalence relations: Some basic considerations. En S. C. Hayes \& L. J. Hayes (Eds.) Understanding verbal relations. The second and third international institute on verbal relations. (pp. 15-27). Reno, Nevada: Context Press.

Sidman, M. (1994). Equivalence relations and behavior: A research history. Boston, MA: Authors Cooperative.

Sidman, M. (2000). Equivalence relations and the reinforcement contingency. Journal of the Experimental Analysis of Behavior, 74, 127-146.

Sidman, M. \& Tailby, W. (1982). Conditional discrimination vs. matching to sample: An expansion of the testing paradigm. Journal of the Experimental Analysis of Behavior, 37, 5-22.

Stromer, R., Mackay, H., McVay, A. \& Fowler, T. (1998) Written lists as mediating stimuli in the matching-to-sample performances of individuals with mental retardation. Journal of Applied Behavior Analysis, 31, 1-19.

Wulfert, E., \& Hayes, S. C. (1988). Transfer of a conditional ordering response through conditional equivalence classes. Journal of the Experimental Analysis of Behavior, 40, 125-144. 